\section{Liens entre apnées du sommeil et altérations cérébrales évaluées par imagerie chez le sujet âgé}

Claire Andrél,2 $^{1,}$ Gaël Chételat ${ }^{1}$, Géraldine Rauchs ${ }^{2}$
${ }^{1}$ Normandie Univ, UNICAEN, Inserm, U1237, Physiopathologie et imagerie des maladies neurologiques (PhIND Physiopathology and imaging of neurological disorders), Institut «Sang et Cerveau » (Institute Blood and brain) @ Caen-Normandie, Cyceron, 14000 Caen, France. ${ }^{2}$ Normandie Univ, UNICAEN, PSL Université, દPHE, Inserm, U1077, CHU de Caen, GIP Cyceron, NIMH, Boulevard Henri Becquerel, BP 5229, 14074 Caen Cedex 5, France. geraldine.rauchs@inserm.fr
> Le vieillissement de la population s'accompagne d'une augmentation de la prévalence des maladies liées à l'âge, et notamment des démences, dont la maladie d'Alzheimer est la forme la plus commune. Son origine probablement multifactorielle reste mal comprise, et aucun traitement curatif n'est actuellement disponible. Une partie importante des recherches vise à identifier les différents facteurs de risque et de protection de la maladie, dans l'espoir de développer des interventions visant à retarder ou ralentir le déclin cognitif. Dans ce contexte, l'étude du sommeil fait l'objet d'un intérêt croissant. $\varepsilon$ n effet, il est établi que le sommeil joue un rôle majeur pour le fonctionnement de la mémoire, et les études menées principalement chez l'animal montrent qu'il jouerait un rôle dans les processus physiopathologiques de la maladie d'Alzheimer. La maladie d'Alzheimer se caractérise par deux processus neuropathologiques: l'accumulation de peptide $\beta$-amyloïde $(A \beta)$ en plaques extracellulaires et l'accumulation intracellulaire de protéine tau, toutes deux conduisant à une neurodégénérescence progressive. Il a été montré, dans un modèle murin de la maladie, que les niveaux d'A $\beta$ et de protéine tau augmentent à l'éveil, et diminuent lors du sommeil $[1,2]$. Ainsi, le sommeil permettrait l'élimination des métabolites toxiques [3]. Ces résultats suggèrent qu'un sommeil de mauvaise qualité pourrait exacerber les processus neu- rodégénératifs conduisant à la maladie d'Alzheimer. Cependant, peu d'études ont été réalisées chez l'homme à ce jour, si bien que les liens entre qualité du sommeil et intégrité cérébrale restent mal compris.

Les apnées obstructives du sommeil sont caractérisées par une réduction totale (apnées) ou partielle (hypopnées) du débit respiratoire. Elles surviennent suite à une obstruction complète ou partielle des voies aériennes supérieures au cours du sommeil, et engendrent des épisodes d'hypoxie intermittente et une importante fragmentation du sommeil. On estime que 30 à $80 \%$ des personnes âgées souffriraient d'apnées obstructives du sommeil [4], et cellesci seraient associées au déclin cognitif et à un risque accru de développer une maladie d'Alzheimer [5]. Cependant, les mécanismes cérébraux sous-tendant cette association sont mal connus. Notre objectif principal a donc été de préciser les atteintes cérébrales structurales, fonctionnelles et moléculaires associées à la présence d'apnées obstructives du sommeil chez des personnes âgées de plus de 65 ans, en utilisant une approche de neuroimagerie multimodale. Les résultats de cette étude ont été publiés dans la revue JAMA Neurology en mars 2020 [6].

Nous avons inclus 127 participants issus de l'essai clinique randomisé Age-Well [7] (https://silversantestudy.fr). Les participants étaient âgés de plus de 65 ans (âge moyen : $69 \pm 3,9$ ans ; $63 \%$ de femmes) et présentaient des performances cognitives dans les normes. Ils ont bénéficié d'une évaluation neuropsychologique, d'une IRM structurale mesurant le volume de substance grise, d'une tomographie par émission de positons (TEP) couplée au ${ }^{18} \mathrm{~F}$-florbetapir mesurant la perfusion cérébrale (acquisition précoce) et les dépôts amyloïdes (acquisition tardive), et, pour un sous-échantillon de 87 participants, d'une TEP couplée au ${ }^{18} \mathrm{~F}$-fluorodésoxyglucose mesurant le métabolisme cérébral du glucose. Un enregistrement complet du sommeil par polysomnographie a été effectué chez tous les participants, et 86 d'entre eux $(68 \%)$ ont bénéficié d'une nuit d'habituation non prise en compte dans les analyses. Ces enregistrements ont été analysés visuellement selon les critères internationaux de l'Académie américaine de médecine du sommeil (AASM), et un index d'apnées-hypopnées (IAH) a été obtenu pour chaque participant. Cet index correspond au nombre d'évènements respiratoires (apnées et hypopnées) par heure de sommeil. Toutes les données ont été acquises au centre Cyceron (Caen, France) entre 2016 et 2018.

Dans un premier temps, nous avons caractérisé les atteintes cérébrales associées à la présence d'apnées obstructives du sommeil. Sur la base de I'IAH, les participants ont été classés comme négatifs ( $\mathrm{AH}<15 ; 31$ participants) ou positifs ( $I A H \geq 15 ; 96$ participants) pour la présence d'apnées obstructives du sommeil. Ces deux groupes ont été com- 
parés pour chaque modalité d'imagerie cérébrale, en prenant en compte l'âge, le sexe, le niveau d'éducation, l'indice de masse corporelle, la prise de médicaments impactant la qualité du sommeil, et la présence de l'allèle $\varepsilon 4$ du gène de l'apolipoprotéine $\varepsilon$, principal facteur de risque génétique de la maladie d'Alzheimer. Les résultats indiquent que les sujets âgés souffrant d'apnées obstructives du sommeil présentent une charge amyloïde plus importante, ainsi qu'une augmentation du volume de substance grise, du métabolisme et de la perfusion cérébrale principalement dans le cortex cingulaire postérieur et le précunéus (Figure 1).

Nous avons ensuite cherché à identifier les mécanismes responsables de ces associations, parmi la fréquence des évènements respiratoires, la sévérité de I'hypoxie intermittente ou de la fragmentation du sommeil associées aux apnées obstructives du sommeil. Pour cela, des régressions pas-à-pas ont été réalisées dans le but de déterminer la variable expliquant le mieux les altérations cérébrales observées. Nous avons montré que la sévérité des épisodes hypoxiques était particulièrement liée à la charge amyloïde.

Enfin, nous avons exploré les liens éventuels avec la cognition et la plainte concernant la qualité du sommeil, grâce à des analyses de corrélations partielles prenant en compte les mêmes covariables que dans l'analyse principale. Aucun lien significatif n'a été retrouvé avec les performances cognitives (attentionnelles, exécutives, de vitesse de traitement des informations, de mémoire de travail, et de mémoire épisodique), la plainte concernant la cognition, les difficultés auto-rapportées de sommeil (mesurées avec l'index de qualité du sommeil de Pittsburgh), ou les symptômes de somnolence diurne excessive (mesurés grâce à l'échelle de somnolence d'Epworth).

Dans l'ensemble, ces résultats suggèrent que la présence d'apnées obstructives du sommeil non traitées est associée à une augmentation de la charge amyloïde dans une région du cerveau typiquement atteinte dans la maladie d'Alzheimer, sans lien direct

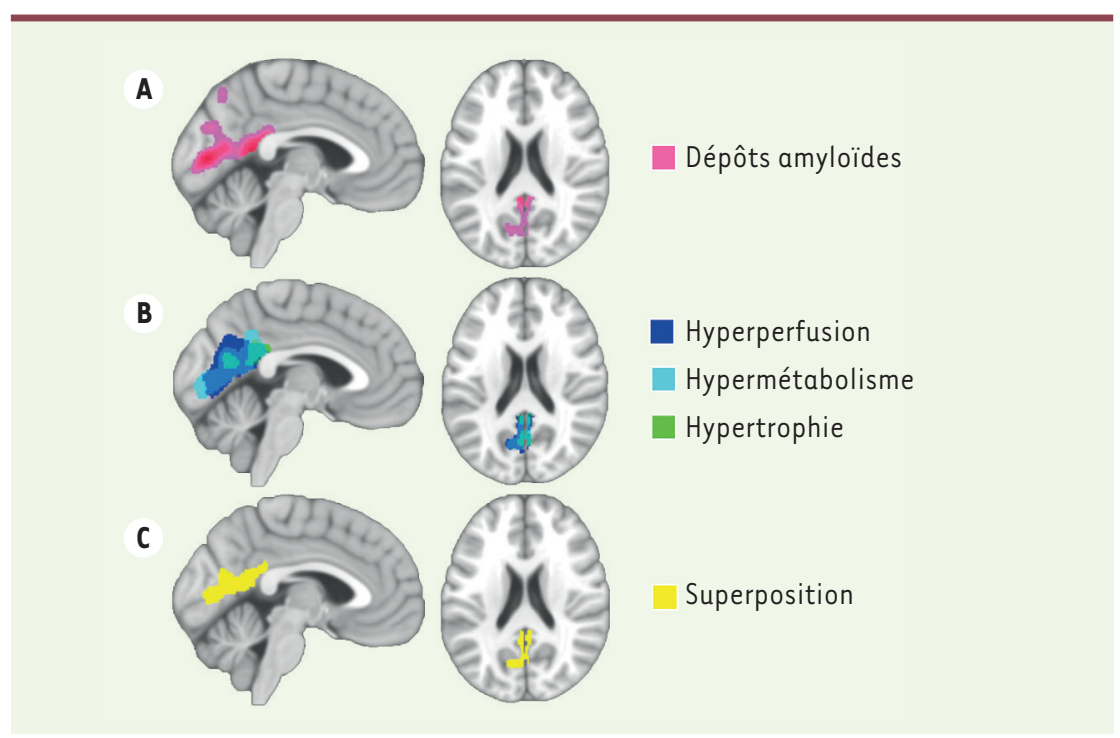

Figure 1. Profil d'atteintes cérébrales associées à la présence d'apnées obstructives du sommeil chez les personnes âgées sans troubles cognitifs. Les participants souffrant d'apnées obstructives du sommeil présentent une augmentation des dépôts amyloïdes ( $A$ ), une hyperactivité neuronale (reflétée par une augmentation de la perfusion et du métabolisme cérébral), et une augmentation du volume de substance grise (B), principalement dans le cortex cingulaire postérieur et le précunéus (c).

avec les symptômes cliniques. Nos résultats sont en faveur d'un rôle délétère de l'hypoxie intermittente, dont il a été montré chez l'animal qu'elle pourrait promouvoir le clivage de I'APP (amyloid precursor protein), augmentant ainsi la production de peptide $A \beta$ [8]. Parallèlement, nous avons montré que l'augmentation de charge amyloïde dans le cortex cingulaire postérieur et le précunéus s'accompagne d'une augmentation du volume de substance grise, de la perfusion et du métabolisme cérébral. Cela pourrait refléter une hyperactivité neuronale et des processus neuro-inflammatoires consécutifs aux apnées obstructives du sommeil, dont on sait qu'ils pourraient favoriser la formation des dépôts amyloïdes $[9,10]$ et précéder les phénomènes d'atrophie et d'hypométabolisme cérébral classiquement observés dans la maladie d'Alzheimer. Ces résultats pourraient également être le reflet d'une plus grande capacité de résilience du cerveau face à différents processus lésionnels chez les participants présentant des apnées obstructives du sommeil, leur permettant de tolérer la charge amyloïde et de maintenir des performances cognitives dans les normes. Des études longitudinales sont nécessaires, notamment pour déterminer si le traitement des apnées obstructives du sommeil chez les personnes âgées permet de ralentir l'accumulation des dépôts amyloïdes et de réduire le risque de déclin cognitif. $\diamond$

Sleep-disordered breathing and Alzheimer's disease biomarkers in older adults

\section{LIENS D'INTÉRÊT}

Les auteures déclarent n'avoir aucun lien d'intérêt concernant les données publiées dans cet article.

\section{RÉFÉRENCES}

1. Kang J, Lim MM, Bateman RJ, et al. Amyloid- $\beta$ dynamics are regulated by orexin and the sleep-wake cycle. Science 2009 ; 326 : 1005-7.

2. Holth JK, Fritschi SK, Wang C, et al. The sleep-wake cycle regulates brain interstitial fluid tau in mice and CSF tau in humans. Science $2019 ; 363: 880-4$. 


\section{RÉFÉRENCES}

3. Xie L, Kang H, Xu Q, et al. Sleep drives metabolite clearance from the adult brain. Science 2013 ; 342 : 373-7.

4. Senaratna ( V, Perret JL, Lodge CJ, et al. Prevalence of obstructive sleep apnea in the general population: a systematic review. Sleep Med Rev 2017 ; 34 : 70-81.

5. Leng Y, McEvoy CT, Allen IE, et al. Association of sleepdisordered breathing with cognitive function and risk of cognitiveimpairment: a systematic review and meta-analysis. JAMA Neurol 2017 ; 74 : 1237-45.
6. André C, Rehel S, Kuhn $\varepsilon$, et al. Association of sleep-disordered breathing with Alzheimer disease biomarkers in community-dwelling older adults: a secondary analysis of a randomized clinical trial. JAMA Neurol 2020 ; e200311.

7. Poisnel G, Arenaza-Urquijo $\varepsilon$, Collette F, et al. The age-well randomized controlled trial of the medit-ageing European project: effect of meditation or foreign language training on brain and mental health in older adults. Alzheimer's Dement Transl Res Clin Interv 2018 ; $4: 714-23$
8. Li L, Zhang X, Yang D, et al. Hypoxia increases Abeta generation by altering beta- and gammacleavage of APP. Neurobiol Aging 2009 ; 30 : 1091-8.

9. Bero AW, Yan P, Roh JH, et al. Neuronal activity regulates the regional vulnerability to amyloid- $\beta$ deposition. Nat Neurosci 2011 ; $14: 750-6$.

10. Kinney JW, Bemiller SM, Murtishaw AS, et al. Inflammation as a central mechanism in Alzheimer's disease. Alzheimer's Dement Transl Res Clin Interv $2018 ; 4: 575-90$. 